INPLASY

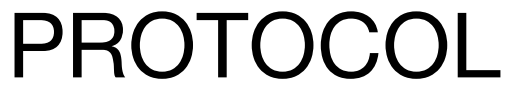

To cite: Xiao et al. The diagnostic test accuracy of ultrasound for hemosiderin deposition of hemophilic arthropathy: A protocol for systemic review and metaanalysis. Inplasy protocol 2020110006. doi: 10.37766/inplasy2020.11.0006

Received: 02 November 2020

Published: 03 November 2020

Corresponding author:

Xuemei Jiang

only6612@163.com

Author Affiliation:

Affiliated Hospital of Shandong University of traditional Chinese Medicine

Support: None.

Review Stage at time of this submission: Formal screening of search results against eligibility criteria.

Conflicts of interest: None.

\section{The diagnostic test accuracy of ultrasound for hemosiderin deposition of hemophilic arthropathy: A protocol for systemic review and meta-analysis}

Xiao, Y1; Jiang, X2; He, H³; Yao, J4; Fang, Y5; Wang, J6.

Review question / Objective: The Ultrasound is recommended as the preferred method for screening and follow-up of hemophilic arthropathy by 2020 World Federation of Hemophilia. However, ultrasound detection of hemosiderin deposition is still controversial, and the guidelines are also ambiguous.

Condition being studied: Hemophilia arthropathy is the most common complication of hemophilia. The basic cause of hemophilic arthropathy is intra-articular hemorrhage, and the main cause of disease progression is hemosiderin deposition. Repeated joint bleeding can lead to a large amount of hemosiderin deposition in the joint cavity, resulting in synovial inflammation. Continuous deposition of large amounts of hemosiderin can cause articular cartilage damage, and then cause irreversible bone destruction, leading to hemophilic arthritis. In the late stage, the joint deformity and disability were caused.

INPLASY registration number: This protocol was registered with the International Platform of Registered Systematic Review and Meta-Analysis Protocols (INPLASY) on 03 November 2020 and was last updated on 16 November 2020 (registration number INPLASY2020110006).

\section{INTRODUCTION}

Review question / Objective: The Ultrasound is recommended as the preferred method for screening and followup of hemophilic arthropathy by 2020 World Federation of Hemophilia. However, ultrasound detection of hemosiderin deposition is still controversial, and the guidelines are also ambiguous.

Condition being studied: Hemophilia arthropathy is the most common complication of hemophilia. The basic 
cause of hemophilic arthropathy is intraarticular hemorrhage, and the main cause of disease progression is hemosiderin deposition. Repeated joint bleeding can lead to a large amount of hemosiderin deposition in the joint cavity, resulting in synovial inflammation. Continuous deposition of large amounts of hemosiderin can cause articular cartilage damage, and then cause irreversible bone destruction, leading to hemophilic arthritis. In the late stage, the joint deformity and disability were caused.

\section{METHODS}

\section{Participant or population: Hemophilia.}

Intervention: Ultrasonic examination.

Comparator: Magnetic resonance imaging.

Study designs to be included: Randomized controlled trial.

Eligibility criteria: (1) Research object: Confirmed or suspected diagnosis of hemophilia based on World Federation of Hemophilia (WFH) classification. (2) The association between imaging technique and the diagnosis of hemophilia patients. (3) Study of imaging diagnostic test for evaluating hemosiderin deposits in hemophilic arthropathy (4) Purposes of literatures was to evaluated hemosiderin deposition in the joint of hemophilic arthropathy by magnetic resonance imaging (MRI) and ultrasound (US) . (5) Diagnostic methods of evaluation: US. (6) Gold standard: MRI. (7) Outcome indicators: false positive (FP), true positive (TP), false negative (FN), true negative (TN). The sensitivity, specificity and likelihood ratio can be calculated with the above data. (8) The published language, age, gender, race and etiology was not limited, and regardless of allocation hidden or blinded.

Information sources: We searched PubMed, Web of Science, Google Scholar, The Cochrane Library, EMBASE, China Biology Medicine disc, China National
Knowledge Infrastructure, Chinese Medical Current Contents, Chinese Science Citation Database and Wanfang database up to October 1, 2019.

Main outcome(s): UItrasound can dynamically and real-time observe the early joint lesions of hemophilia, including joint effusion, intra-articular synovial hyperplasia, synovial vascular proliferation, late irreversible cartilage damage and bone destruction. MRI is the gold standard for the diagnosis of hemophilic arthropathy at present. It has a good consistency with ultrasound in the diagnosis of synovial hyperplasia and joint effusion, while it is more comprehensive and accurate for bone and cartilage damage than ultrasound. However, ultrasound has obvious advantages in synovial vascular hyperplasia, and MRI should be detected by invasive contrast-enhanced examination. However, for hemosiderin deposition, MRI examination is obviously superior to ultrasound examination, and there is no uniform ultrasonic sign of hemosiderin deposition on the sonogram.

Quality assessment / Risk of bias analysis: In this study, QUADAS-2 (Quality Assessment of Diagnostic Accuracy Studies 2) [12]was used to evaluate the risk of bias and applicability of primary diagnostic accuracy studies included in the meta-analysis. Quadas-2 includes: patient selection, index test, reference standard, flow and timing. According to the answers of "yes", "no" or "uncertain" to the relevant landmark questions included in each part, the risk level of bias can be determined as "low", "high" or "uncertain". If the answer to all the landmark questions in a range is "yes", then it can be rated as low risk of biases; if one of the answers to all the information questions is "no", then it can be rated as the high possibility of various biases. If there is a possibility of bias, the evaluator must then use the guidelines developed in stage 2 to determine the risk of bias. The classification of "uncertainty" is that there is no detailed content in literature, which makes it difficult for evaluators to make judgment. This kind of 
literature can only be used when the reported data is insufficient.

Strategy of data synthesis: We used an exact binomial rendition of the bivariate mixed-effects regression model[13, 14] to estimate mean logit sensitivity and specificity with their standard error and $95 \%$ Cls. And construct a summary receiver operating curve (SROC) for US diagnosis of hemosiderin deposition sensitivity and specificity on the curves and a $95 \%$ confidence contour ellipsoid (twodimensional $\mathrm{Cl}$ ).

Subgroup analysis: According to the characteristics of the study, representative characteristics were selected and subgroup analysis was conducted to observe the consistency between the results of subgroup analysis and metaanalysis, so as to determine whether each factor has an important impact on the results of the combined effect. The results of subgroup analysis are relatively reliable, if the sample size of subgroup analysis is large, which can better support or overturn the comprehensive effect of meta-analysis.

Sensibility analysis: The implementation methods of sensitivity analysis include three methods, such as changing the analysis model, excluding the literature one by one, and cutting and supplementing method. When the results of sensitivity analysis are consistent, indicating the results are relatively stable and robust. It can be considered that the research results are relatively reliable. On the contrary, When the results of sensitivity analysis are inconsistent, it indicates that the results are not robust and should be treated with caution. In depth analysis may be needed to explore the sources of these key factors.

Country(ies) involved: China.

Keywords: hemophilic arthropathy, synovial hemosiderin deposition, ultrasound, diagnostic, accuracy, meta-analysis, protocol.

Contributions of each author:
Author 1 - Yanyan Xiao - Author 1 drafted the manuscript.

Author 2 - Xuemei Jiang.

Author 3 - Hongan He.

Author 4 - Junpeng Yao.

Author 5 - Yunhai Fang.

Author 6 - Jie Wang. 